\title{
Fronto-Tectal White Matter Connectivity Mediates Facilitatory Effects of Non-invasive Neurostimulation on Visual Detection
}

\author{
Romain Quentin ${ }^{1}$, Lorena Chanes ${ }^{1}$, Raffaella Migliaccio ${ }^{1}$, Romain Valabrègue ${ }^{4}$, and Antoni \\ Valero-Cabré ${ }^{1,2,3,{ }^{*}}$ \\ ${ }^{1}$ Université Pierre et Marie Curie, CNRS UMR 7225-INSERM UMRS 975, Centre de Recherche \\ de l'Institut du Cerveau et de la Moelle épinière (ICM), Paris, France.
}

2Laboratory for Cerebral Dynamics Plasticity \& Rehabilitation, Boston University School of Medicine, Boston, USA.

${ }^{3}$ Cognitive Neuroscience and Information Technology Research Program, Open University of Catalonia (UOC), Barcelona, Spain.

${ }^{4}$ Centre de Neuroimagerie de Recherche (CENIR), Centre de Recherche de l'Institut du Cerveau et de la Moelle (ICM), Paris, France.

\begin{abstract}
The causal ability of pre-target FEF activity to modulate visual detection for perithreshold stimuli has been recently demonstrated in humans by means of non-invasive neurostimulation. Yet in spite of the network-distributed effects of these type of techniques, the white matter (WM) tracts and distant visual nodes contributing to such behavioral impact remain unknown. We hereby used individual data from a group of healthy human subjects, who received time-locked pulses of active or sham Transcranial Magnetic Stimulation (TMS) to the right Frontal Eye Field (FEF) region, and experienced increases in visual detection sensitivity. We then studied the extent to which interindividual differences in visual modulation might be dependent on the WM patterns linking the targeted area to other regions relevant for visuo-attentional behaviors. We report a statistically significant correlation between the probability of connection in a right fronto-tectal pathway (FEFSuperior Colliculus) and the modulation of visual sensitivity during a detection task. Our findings support the potential contribution of this pathway and the superior colliculus in the mediation of visual performance from frontal regions in humans. Furthermore, we also show the ability of a TMS/DTI correlational approach to contribute to the disambiguation of the specific long-range pathways driving network-wide neurostimulatory effects on behavior, anticipating their future role in guiding a more efficient use of focal neurostimulation.
\end{abstract}

\section{Keywords}

Frontal Eye Field; Cortico-tectal projections; Visuo-Spatial Attention; Neuroanatomy; Transcranial Magnetic Stimulation; Diffusion Tensor Imaging

\footnotetext{
(C) 2013 Published by Elsevier Inc.

*Corresponding author: Dr. Antoni Valero Cabré, MD PhD, CNRS-INSERM-Université Pierre et Marie Curie. ICM, Equipe Cognition, Neuro-imagerie et maladies du Cerveau; Groupe de Dynamiques Cérébrales, Plasticité et Rééducation. Hôpital de la Salpêtrière, 47-boulevard de l'Hôpital, 75651 Paris Cedex 13, France. avalerocabre@gmail.com; avalero@bu.edu.

Publisher's Disclaimer: This is a PDF file of an unedited manuscript that has been accepted for publication. As a service to our customers we are providing this early version of the manuscript. The manuscript will undergo copyediting, typesetting, and review of the resulting proof before it is published in its final citable form. Please note that during the production process errors may be discovered which could affect the content, and all legal disclaimers that apply to the journal pertain.
} 


\section{INTRODUCTION}

Visual systems translate information from the real world into sophisticated bioelectrical patterns, which can be used thereafter to build a neural representation of our environment. It is well known that such function is strongly modulated by bilaterally distributed frontoparietal networks in charge of orienting attention to specific regions of the space, facilitating the detection and discrimination of visual stimuli (Yeshurun \& Carrasco 1999; Carrasco et al. 2000; Carrasco et al. 2001; Cameron et al. 2002). Such operations are essential for the selection of behaviorally crucial targets to be attended in a world rich in distractors, without being overwhelmed by numerous and divers sources of information.

Fronto-parietal visuo-attentional networks are classically divided into a bilateral dorsal system, linking the Frontal Eye Fields (FEF) and the Intraparietal Sulcus (IPS), involved in the orienting of attention in space (Beauchamp et al. 2001; Corbetta \& Shulman 2002; Shulman et al. 2010; Chica et al. 2011), and a ventral right-lateralized system between the middle and the Inferior Frontal Gyrus (MFG/IFG), and the Temporo-Parietal Junction (TPJ), responsible for the reorientation of attention during unexpected events (Corbetta \& Shulman 2002; Shulman et al. 2010; Chica et al. 2011). Importantly, neuroimaging data (Nobre et al. 1997; Petit et al. 2009), non-invasive neurostimulation evidence (Hilgetag et al. 2001; Thut et al. 2005; Grosbras \& Paus 2003), and clinical observations (Bartolomeo et al. 2012) strongly support a right hemisphere dominance in visuo-spatial attention, and demonstrate the ability of such right-hemisphere systems to influence visual perception for targets in both visual hemifields (Grosbras \& Paus 2003, Chanes et al. 2012).

White matter (WM) connections established between nodes of this network have been thoroughly studied and remain essential to understand its contributions to spatial attention and perception. Intracortical microstimulation and tracing studies carried out in the non human primate brain have shown that the FEF is highly connected to the superior and ventral portions of the parietal lobe and to caudal regions of the superior temporal cortex (Huerta et al. 1987; Stanton et al. 1995). Similarly in humans, this network is underlain by a rich set of anatomical WM projections which in homology to non human primates have been recently identified as the three branches of the Superior Longitudinal Fasciculus (Thiebaut de Schotten et al. 2012), linking key regions of the frontal and the posterior parietal lobe (Umarova et al. 2010). Finally, connections of fronto-parietal systems with subcortical structures such as the pulvinar nucleus of the thalamus and the superior colliculus (SC) in the midbrain are also important and contribute to both overt and covert attentional deployment (Shipp 2004).

Engaged exogenously (i.e., by reflexively capturing attention) or guided endogenously (i.e., according to feature-based instructions), these circuits have the ability to modulate the gain of retinal incoming signals to cortical (Reynolds et al. 2000; Reynolds \& Robert Desimone 2003; Reynolds \& Chelazzi 2004) and subcortical structures (Wurtz \& Goldberg 1972; Gattass \& R Desimone 1996; O'Connor et al. 2002; Schneider \& Kastner 2009), and impact visual processing. Indeed, studies in non-human primates have proven the potential of FEF microstimulation, alone or in combination with bottom-up visual inputs to modulate activity in visual areas (Reynolds \& Chelazzi 2004; Ekstrom et al. 2009) and influence visual performance (Moore \& Fallah 2004). Similarly in humans, Transcranial Magnetic Stimulation (TMS), a tool which induces pattern-dependent local and transynaptic effects through long-range connectivity (Valero-Cabré et al. 2005; Valero-Cabré et al. 2007) has shown the ability to elicit in the FEF and IPS regions brief (Grosbras \& Paus 2003; Grosbras \& Paus 2002; Ruff et al. 2006; Silvanto et al. 2006) and transient (Hilgetag et al. 2001; Thut et al. 2005) modulations of visual behaviors. 
In a recent report, we demonstrated that single-pulse TMS stimulation delivered to the right FEF had the ability to improve the detection but not the discrimination of low-contrast nearthreshold visual stimuli (Chanes et al. 2012). This result strongly supports the notion that vision can be non-invasively manipulated and enhanced in humans. However the underlying circuitry linking the targeted regions, the right FEF, and other brain locations contributing to such visual ameliorations are not easy to disambiguate on the basis of differences in the behavioral patterns recorded under the causal influence of neurostimulation. Using a hypothesis-driven approach, we correlated individual MRI diffusion data (Behrens et al. 2007) from a set of four anatomically plausible tracts emerging from the stimulated right FEF and known to be involved in the orienting of spatial attention, and visual performance outcomes modulated by TMS. A preceding Tract-Based Spatial Statistics (TBSS) datadriven analysis was employed in an attempt to identify WM voxel clusters with diffusion measures correlated to visual performance outcomes. Overall, we aimed to identify the WM pathways which would best explain individual effect differences in visual sensitivity for our population of right FEF neurostimulated participants (Chanes et al. 2012) that could be most likely associated with our patterns of behavioral effects. We hypothesized that the WM pathways significantly correlated with neurostimulation-driven outcomes would involve brain sites linked to the FEF, with processing features and abilities compatible with the characteristics of the modulated visual behaviors.

\section{MATERIAL AND METHODS}

\subsection{Participants}

The TMS data used in the current manuscript were extracted from two experiments of a recently published behavioral-TMS data set (Chanes et al. 2012). This study included 13 human subjects ( 5 males and 8 females; mean age, $23.8 \pm 3$ years; minimum age, 18 years; maximum age, 28 years). All these participants provided informed written consent and were compensated for their participation. They all participated voluntarily, and were naïve to both, the purpose of the experiment and the uses of TMS. The protocol was reviewed by the Inserm (Institut National de la Santé et la Recherche Scientifique) ethical committee and approved by an Institutional Review Board (CPP Ile de France 1).

\subsection{Behavioral paradigm and TMS stimulation}

The visual paradigm used in both experiments worked as follows. A fixation point was displayed in the center of the screen, along with three black square boxes, one central and two lateral ones. The target consisted of a Gabor stimulus, which could appear at the center of one of two lateral boxes for a brief period of time $(33 \mathrm{~ms})$. The Gabor lines were tilted $1^{\circ}$ to $10^{\circ}$ to the left or to the right (corresponding $0^{\circ}$ to their vertical orientation). Single TMS pulses were delivered $80 \mathrm{~ms}$ prior to target onset on the right FEF. This region was individually labeled on each individual MRI volume using averaged Talairach coordinates $x=31, y=-2, z=47$ (Paus 1996). This location was confirmed on each participant's MRI native space by a procedure based on the elicitation of saccade preparation delays under the impact of single TMS pulses on this site (Thickbroom et al. 1996; Ro et al. 2002; Grosbras and Paus 2002 and 2003; Chanes et al 2012). In Experiment 1 (pre-target onset TMS pulses alone), after a variable fixation period of time (1000-1500 ms), the central fixation cross became slightly bigger for $66 \mathrm{~ms}$ and following an Interstimulus Interval (ISI) of $233 \mathrm{~ms}$ a target was displayed for $33 \mathrm{~ms}$ within one of the two lateral boxes. Single TMS pulses were delivered on the right FEF 80, 100 or 140 ms prior to the target onset. Active TMS pulses were randomly interleaved by an equal number of sham single pulses delivered by a second TMS coil with its surface located perpendicular to the head surface, next to the right FEF site. The experiment consisted of 600 trials, including 120 target-absent trials. In Experiment 2 (pre-target onset spatial cues combined with single TMS pulses), the paradigm was kept 
identical, except that a peripheral cue consisting in a black dot (1.5 diameter) was presented for $66 \mathrm{~ms}$ in the upper outer corner of one of the two lateral square boxes to orient the attention of the participant to that location (Figure 1). After an identical ISI, a Gabor appeared at the center of the cued (valid trials) or uncued (invalid trials) lateral box. The cue was predictive about the location of the subsequent target $(75 \%$ valid and $25 \%$ invalid trials). For this second experiment, single TMS pulses were delivered on the right FEF 80 ms prior to target onset. This second experiment consisted of 800 trials, including 160 target-absent trials.

In both experiments, participants were first required to determine the orientation of the Gabor lines (categorization task) as fast and as accurately as possible. They were encouraged to respond to every trial within a window of $2000 \mathrm{~ms}$, and forced to guess a response, even when the target was not present or they did not consciously perceive it. Secondly, they were requested to report whether they perceived the Gabor in the left, in the right, or they did not see it (detection task). Categorization performance was analyzed through accuracy (correct grating orientation categorization) and reaction time for correctly reported targets. Perceptual sensitivity (d') and response bias (beta) used in Signal Detection Theory (SDT) served to assess the modulation of visual detection in the second response. Subjects were requested to keep their gaze on the fixation cross throughout the trial. Correct fixation was controlled by an eye-tracker system. Target contrast was adjusted prior and throughout the task so that $\sim 62 \%$ of the displayed targets were reported (detection task) and 65 to $85 \%$ of the correctly reported ones were correctly discriminated (categorization task) (see Chanes et al. 2012 for details).

\subsection{MRI acquisition}

Prior to the TMS study, diffusion tensor MRI scans were obtained in all thirteen participants on a 3 Tesla MRI scanner (Tim Trio, Siemens Healthcare, Erlangen, Germany) located in the CENIR (Centre de Neuro-Imagerie de Recherche) at the Hôpital de la Pitié Salpêtrière, in Paris (France). Using a 12-channel array coil and a maximum gradient strength of $28 \mathrm{mT} /$ $\mathrm{m}$, diffusion weighting was isotropically distributed along 64 directions. Note that high angular resolution of the diffusion weighting directions yields robust probability density estimation by increasing the signal-to-noise ratio and reducing directional bias. One image with no diffusion (b0) was acquired initially and served as an anatomical reference for eddy current corrections. The imaging parameters were repetition time $(\mathrm{TR})=11.000 \mathrm{~ms}$, echo time $(\mathrm{TE})=88 \mathrm{~ms}, \mathrm{~b}=1000 \mathrm{~s} / \mathrm{mm} 2$ and matrix size $=128 \times 128 \times 60$. Each set of images contained 60 contiguous slices with a $2 \mathrm{~mm}$ thickness. Total acquisition time was $12 \mathrm{~min}$ and 30 seconds. This resulted in a tensor for every voxel $\left(2 \times 2 \times 2 \mathrm{~mm}^{3}\right)$ in a slice. The eigenvectors and eigenvalues were computed for every tensor, to constitute the raw data set for tractography analysis. A 3D structural T1-weighted MRI was also acquired for each subject $(\mathrm{TR}=2300 \mathrm{~ms}, \mathrm{TE}=4.18 \mathrm{~ms}, \mathrm{FOV}=256 \mathrm{~mm}$, matrix size $=256 \times 256,176$ sagittal slices with thickness $=1 \mathrm{~mm}$ ).

\subsection{Data preprocessing, Tract-Based Spatial Statistics (TBSS) and Tractography}

Data preprocessing-Diffusion tensor imaging (DTI) pre-processing and analyses were performed using the Functional Magnetic Resonance Imaging of the Brain (FMRIB) Software Library (FSL 4.1.6 - www.fmrib.ox.ac.uk/fsi/). Head motion effect and image distortion caused by eddy currents were corrected using affine registration to the reference volume b0 and the Brain Extraction Tool (BET) was applied to remove non-brain tissues. The gradient direction for each volume was corrected using the rotation parameters. Spatial deformation of the DTI due to the susceptibility artifact were corrected with non-linear deformation computed from the diffusion images to match the T1 weighted volume using Freesurfer Software (Freesurfer 5.0.0, http://surfer.nmr.mgh.harvard.edu/). The diffusion 
tensor and the three eigenvalues were computed by fitting a tensor model to correcteddiffusion data using FLS's DTIFIT and resulted in several DTI-based map, including an FA map.

Tract-Based Spatial Statistics-The following steps here were carried out with the TBSS software module (Smith et al. 2006). A non-linear registration was first applied to align the FA map across subjects. These data were then registered for all participants to the $1 \times 1 \times 1 \mathrm{~mm}^{3}$ MNI152 space (McConnell Brain Imaging Center, Montreal Neurological Institute). A common skeleton from the mean FA image representing the core-structure of the WM tract was created. This skeleton was thresholded at a FA value of 0.2. Normalized FA data were then projected onto this skeleton. These FA images were entered into a voxelwise General Linear Model (GLM) analysis. To study the potential correlations between each voxel's FA value and individual visual performance modulation under TMS, we employed as an explanatory covariate in both experiments the visual sensitivity (d') difference between sham vs. real TMS trials in two separate models with participant's age as confounding covariate. The randomized permutation-based nonparametric inference routine was used with 5000 permutations. Finally, Threshold-Free Cluster Enhancement (TFCE) was employed to control for multiple testing. This method avoids the need for an arbitrary initial cluster-forming threshold. Statistical significance threshold was set at $\mathrm{p}<0.05$.

Tractography-As the TBSS approach did not reveal any significant correlations between white matter voxels and TMS visual performance outcomes (see Results, section 3.3 for details), a fiber tracking procedure based on a probabilistic tractography method and the dual-fiber model implemented in FMRIB diffusion (5000 streamlines samples, $0.5 \mathrm{~mm}$ step lengths, curvature threshold $=0.2$ ) (Behrens et al. 2007) was used to identify fibers departing from the TMS stimulated site, the right FEF, specifically projecting to the following 4 selected destinations within the right hemisphere: the Intra-Parietal Sulcus (IPS), the Supra-Marginal Gyrus (SMG), the Temporo-Parietal Junction (TPJ), and the Superior Colliculus (SC). Such limited number of most likely destinations and WM tracts were selected on the basis of their strong anatomical plausibility, their known contributions to the modulation of attentional orienting and visual perception and their hypothesized implication in network-wide modulations of visual behaviors from the right FEF site. This hypothesis-driven tractography analysis was performed in each participant's native diffusion space. The right FEF and the above mentioned 4 distant right hemisphere regions were used respectively as seed and termination masks in four independent tractography analyses. All tracts were in the end overlaid on the anatomical MRI volume, visually checked for consistency with regards to the known human brain anatomy, and normalized using the MNI template. A $15 \%$ threshold of the maximum voxel intensity of each individual fasciculus was applied to remove extraneous tracts, and used for their presentation in figures.

ROI delineation-Using the Statistical Parametric Mapping software (SPM8, http:// www.fil.ion.ucl.ac.uk/spm) running on Matlab 7.11.0 (Math-Works, Natick, MA), T1weighted images were registered linearly to the b0 images. Each region-of-interest (ROI) was then created on the T1 Montreal Neurological Institute (MNI) standard brain and denormalized in the native diffusion space of each subject. The anatomical localization of each subject's ROIs was verified on T1-weighted images. Anatomical verification on structural images allowed a more accurate localization of ROIs and tracts than in the native diffusion space (Basser et al. 2000). A sphere of $1 \mathrm{~cm}$ radius centered on the TMS targeted right FEF coordinates was used as frontal ROI. The size of this sphere was adapted on the basis of an estimated spatial resolution for TMS pulses using a standard $70 \mathrm{~mm}$ figure-ofeight coil of 1.5-2.0 cm² (Valero-Cabré et al. 2005; Thielscher \& Kammer 2004). For the IPS, TPJ and SMG ROIs, $0.425 \mathrm{~cm}$ radius spheres centered on the Talairach (IPS: $\mathrm{x}=16, \mathrm{y}=$ 
$-63, \mathrm{z}=47$, TPJ: $\mathrm{x}=51, \mathrm{y}=-51, \mathrm{z}=26$ ) or MNI coordinates (SMG: $\mathrm{x}=54, \mathrm{y}=-37, \mathrm{z}=46$ ) obtained from prior fMRI studies on attentional orienting networks and probed in TMS studies (Kincade et al. 2005; Chica et al. 2011) were used. The SC ROI was drawn on the T1 MNI template $(0.5 \mathrm{~mm}$ voxel size) on 12 consecutive axial sections. The radius of the spheres for IPS, SMG, and TPJ was chosen to match the volume of the SC ROI.

\subsection{White matter bundles-behavioral correlations}

Differences among participants in terms of average trajectory count between pairs of ROIs were compared by means of the number of paths from the seed region (in the targeted coordinates of the right FEF) reaching the destination areas within the right hemisphere (IPS, SMG, TPJ and SC). In order to avoid dependence from the number of samples launched by the algorithm, the number of paths was divided by the volume in voxels of the departure ROI. This calculation provided for each fasciculus an estimation of the connection probability between the two regions. By dividing this value by the number of trajectories launched in each seed voxel, i.e. 5000, a probability of connection value between 0 and 1 (and then multiplying by 100 to present it as percentage) can be easily calculated. A Wilcoxon signed-rank test was used to test the differences between the four pathways. This difference was considered significant for a $\mathrm{p}$ value $<0.05$.

We focused particularly on the correlation of visual performance parameters (d') having shown in our prior study statistically significant group modulations under the combined effect of spatial cues and single TMS pulses delivered to the FEF (Experiment 2), as this was the only data set in which attentional orientation was explicitly manipulated by means of spatial cues (Chanes et al. 2012). As in a repeated measures ANOVA, the significant double interaction included the factors TMS pulse type (sham, real) and cue validity (valid, invalid) but excluded target location (right, left), we assumed that the facilitatory effects driven by right FEF TMS spanned bilaterally to both visual hemifields (Grosbras \& Paus 2003). Accordingly, perceptual sensitivity modulations for both hemifields were grouped for further correlational analyses (Chanes et al. 2012). Improved visual detection performance under the effects of single-pulse TMS was presented for each subject in terms of perceptual sensitivity (d') levels under the effects of interleaved active vs. sham TMS pulses. The difference between these two TMS conditions was calculated to obtain the relative visual sensitivity gain $\left(\Delta d^{\prime}\right)$ induced in active TMS trials with respect to sham TMS trials. Spearman's rank correlation coefficients were calculated using statistical analysis software (JMP 8.0, SAS, Cary, NC USA). Bonferroni post-hoc correction, which lowers the alpha value to account for the number of comparisons being performed, was used to avoid spurious positives (uncorrected $\mathrm{p}<0.05$; corrected $0.05 / 4$ tracts $\mathrm{p}<0.0125$ ). To provide a convincing proof of the robustness of our statistically significant correlation, a permutation test (Groppe et al. 2011) based on Spearman's rank correlation coefficient with 5000 permutations was applied. The null hypothesis of the permutation test is that every possible order of a given observation is as likely as a correlation obtained by chance. Finally, to further ensure the correlation of a given pathway with behavioral outcomes, independent of the influence of the other bundles considered in the analyses, we implemented a multiple linear regression analysis based on the General Linear Model (GLM) implemented in Matlab (7.11.0 Math-Works, Natick, MA). The GLM expresses behavioral data as a linear combination of a set of explanatory variables, such in our case, the WM pathway's probability of connection. This test was considered significant for a $p$ value $<0.05$.

\subsection{Anatomical description of significantly correlated pathway}

The anatomical trajectories of the significantly correlated pathways were described in detail. To this end, tractography trajectories were normalized to the standardized MNI template and their group average calculated (final image resolution of $2 \times 2 \times 2 \mathrm{~mm}^{3}$ ). As indicated 
above, in order to remove extraneous tracts, a threshold of $15 \%$ of the maximum voxel intensity for each individual fasciculus was applied. This threshold was calculated for each individual participant before the tracts were averaged across participants in MNI space and thus it played no role or influenced in any way the correlations between white matter and behavioral outcomes; it was simply used for figure presentation purposes. Finally, a detailed and highly magnified description of each pathway' trajectory was performed on serial axial slices for each subject, and for the group average pathway, under the supervision of an experienced neuroradiologist and a neuroanatomist. The MRIcroGL software (http:// www.mccauslandcenter.sc.edu/mricrogl) was used for glass brain illustrations.

\section{RESULTS}

\subsection{Individual detection sensitivity modulation induced by TMS}

As reported elsewhere at a group level, pre-target onset TMS pulses combined with valid spatial cues in those subjects able to efficiently capture and orient attention in space increased visual detection sensitivity (d') for active as compared to sham TMS. No differences between active and sham TMS were observed for invalid trials, in which the cue incorrectly signaled the location of the target. Finally, no modulations were neither observed for the categorization task (see Experiment 2, Chanes et al. 2012). Interestingly however, the 13 participants demonstrated some level of variability in the magnitude and direction of the above-mentioned visual sensitivity modulations $\left(\Delta d^{\prime}\right)$. More specifically, 1 participant seemed to experience no TMS driven effects, 2 participants displayed an apparent deterioration of their visual performance, and the magnitude of visual sensitivity improvements (d'TMS - d'sham TMS) experienced by the remaining 10 subjects oscillated greatly between $\Delta d$ ' values of 0.06 and 0.43 (see Figure 2 for details).

\subsection{Hypothesis-free approach: Tract-based Spatial Statistics (TBSS) results}

We tested the correlations between voxel-wise analysis of FA and TMS visual sensitivity modulations $\left(\Delta d^{\prime}\right)$ using for Experiment 1, data of sham and real TMS trials including exclusively pulses delivered $80 \mathrm{~ms}$ pre-target; and for Experiment 2, data from sham and real TMS trials encompassing TMS pulses delivered $80 \mathrm{~ms}$ pre-target combined with peripheral predictive cues. No significant correlations between the FA and behavioral performance, fully corrected for multiple comparisons across space, were found for any of these two behavioral datasets ( $p>0.05$ ). An additional test for such correlations with a lower $\mathrm{p}$ value $(\mathrm{p}<0.001)$ using non-corrected correlations map did not yield any significant result either. In sum, in our data sets, the TBSS approach did not capture any significant correlation between white matter anisotropy measures and visual performance modulations.

\subsection{Hypothesis-driven approach: Identification of relevant white matter pathways}

For our group of 13 subjects, we tracked a total of 4 right hemisphere tracts, all seeded in the coordinates of the right FEF region targeted in our TMS experiment: FEF-IPS, FEF-SMG, FEF-TPJ, and FEF-SC (see Figures 2 and 3). As indicated in the methods section this limited set of potential connections was selected on the basis of their anatomical plausibility, their contribution to attentional orienting and visual perception and their hypothesized implication in network-wide modulations of visual behaviors from the right FEF site. Our results show that the right FEF-IPS and FEF-SC white matter tracts shared similar average trajectory count values $(2.48 \pm 5.88$ vs. $2.77 \pm 3.05$, $\mathrm{p}=0.37)$. In contrast, the FEF-TPJ $(0.25 \pm 0.53)$ tract appeared as significantly less connected than the FEF-IPS $(\mathrm{p}=0.013)$ and the FEF-SC ( $\mathrm{p}=0.013)$. Finally, the FEF-SMG $(0.44 \pm 0.56)$ appeared as not statistically differently connected than the FEF-IPS ( $\mathrm{p}=0.41$ ) or the FEF-TPJ $(\mathrm{p}=0.31)$, but significantly less connected than the FEF-SC white matter tract $(\mathrm{p}=0.008)$. This across-tract statistical comparison should however be taken cautiously as connection probability may depend on 
the tract length and curvature, factors that cannot be easily accounted for in our study. Note that such low values of average trajectory count, which are also common in other probabilistic tractography studies are explained by sharp decreases in anisotropy and high levels of noise affecting diffusion signal as the launched trajectories approach cortical regions (Behrens et al. 2007).

\subsection{White matter bundles-behavioral correlations}

We then computed the correlations between the individual behavioral effects expressed in terms of perceptual sensitivity differences $\left(\Delta \mathrm{d}^{\prime}\right)$ for the combination of a pre-target TMS pulse and valid spatial cues with the individual average trajectory count of our 4 pre-selected WM pathways (Figures 2 and 3). The only statistically significant correlation was found between the FEF-SC and improvements induced by the combination of TMS and valid cues for bilateral visual stimuli $(\rho=-0.80, p=0.001)$. Interestingly, this correlation was negative, i.e., the higher the fronto-tectal probability of connection, the lower the magnitude of the TMS-driven visual facilitatory effects or vice versa (Figure 3). As indicated above, no significant correlations were found for any of the remaining three white matter bundles considering either corrected or uncorrected $\mathrm{p}$ threshold significance values (FEF-IPS: $\rho=0.033, p=0.915$, FEF-SMG: $\rho=-0.214, p=0.482$, FEF-TPJ: $\rho=-0.131, p=0.654$ ) (Figure 4). For the only pathway (FEF-SC) significantly correlated to TMS driven visual sensitivity measures, the non-parametric permutation test confirmed the robustness of the result $(\mathrm{p}=0.002)$.

A multiple linear regression analysis using the General Linear Model (GLM) with the statistically significant pathway as explanatory variable and the non-significant pathways and participant's age as confound regressor was tested. To rule out if other measurable factors could have bearing on the current behavioral results, skull-thickness at the stimulation site, straight distance from the skull to the FEF site, the estimated magnetic field strength in the targeted area, TMS stimulation intensity ultimately used to stimulate each subject and the individual motor thresholds as a measure of cortical excitability, were also used as covariables in the GLM model. This analysis also confirmed that FEF-SC pathway was significantly correlated with the reported visual behavioral outcomes $(\mathrm{t}=-4.66, \mathrm{p}$ $=0.005, \mathrm{df}=3$ ).

We also verified posthoc a potential interaction between the identified fronto-tectal pathway and the effects of cue-driven attentional orienting in absence of effective TMS stimulation on visual sensitivity. This was explored by considering only trials (data from Experiment 2) including visuo-spatial attentional cues combined with interleaved sham TMS pulses (sham TMS validly cued trials - invalidly cued trials). The correlations for such behavioral outcomes proved once more non-statistically significant $\left(r^{2}=0.089, p=0.320\right)$, suggesting that cue-driven attentional orienting per se could not explain the significant correlation found between TMS modulated visual performance outcomes and the probability of connection for the right FEF-SC pathway. Finally, we tested posthoc a potential correlation between the right FEF-SC pathway and the effects of isolated pre-target onset TMS pulses in absence of visuo-spatial cues (data from Experiment 1). Once more, no significant correlation was found $\left(\mathrm{r}^{2}=0.045, \mathrm{p}=0.505\right)$, suggesting that it was rather the combined effects of both events, attentional orienting induced by valid spatial cues and right FEF TMS stimulation, and not the isolated effects of either one or the other, which were influenced by the previously identified fronto-tectal WM tract.

\subsection{Anatomical description of the fronto-tectal pathway trajectory}

The fronto-tectal (FEF-SC) pathway was the only tract in our hypothesis-based correlational approach holding statistically significant correlations with visual detection facilitatory 
effects. According to our mean tract calculations this tract, defined as departing from the FEF and projecting to the SC, progressed within the corona radiata towards the upper part of the anterior limb of the internal capsule. Then, in an almost horizontal trajectory, placed itself in the genu of the internal capsule to reach the thalamus. The limited resolution of our DTI anatomical images did not allow us to define the intra-thalamic trajectory of those fibers with sufficient precision. Nonetheless, in agreement with previous anatomical description of a compatible fronto-tectal tract in non-human primates explored by tracer injection (Leichnetz et al. 1981), fibers could have progressed within the internal medullary lamina and paralaminar regions of the thalamus. From the posterior portion of the thalamus, fibers reached the brachium of the superior colliculus to terminate in the upper tectum (Figure 5).

\section{DISCUSSION}

In view of the network-distributed effects of TMS on neural systems and their role in performance modulations (Valero-Cabré et al. 2005, Valero-Cabré et al. 2007), we hypothesized that WM connectivity could be among the important factors explaining interindividual behavioral variability to TMS. We used a combination of TMS and DTI recordings to address, if specific WM fasciculi might have bearing on the individual patterns and magnitudes of visual performance ameliorations induced by the stimulation of the right FEF region. A correlational approach could help to disambiguate which WM tracts and distant visuo-attentional regions were most likely contributing to the propagation of TMS effects from the stimulated cortical site and the reported visual sensitivity improvements. Our final results based on FSL's dual fiber model show that out of a set of the four most plausible right hemisphere tracts included in our correlational study (see methods for details on selection criteria), only a trans-thalamic fronto-tectal projection (FEF-SC) emerged as a WM pathway, whose probability of connection was significantly correlated to the impact of active TMS combined with spatial cues, and their effects on the modulation of visual sensitivity $(\Delta d ')$.

This hypothesis-driven approach was preceded however by a data-driven method based on a whole brain voxel-wise correlation method, TBSS, which for both, corrected and uncorrected analyses, failed to unearth WM clusters with FA measures significantly correlated to visual performance. Although we cannot rule out additional explanations, the TBSS approach might have been limited to detect significantly correlated clusters related to a fronto-tectal pathway as the one we finally identified, for at least two reasons: first, the FA measure is based on a single diffusion tensor (Basser \& Pierpaoli 1996), and may be less sensitive in voxels with more than a single fiber direction than the FSL's dual fiber approach (which models two fibers per voxel) for the fronto-tectal tract. Indeed, the FA value depends on a large set of structural and physiological variables, like the number, diameter and myelination level of axons, but is also influenced by the intra-voxel orientational dispersion (Jones et al. 2012). Second, the mean FA skeleton used in TBSS employs the centers of fiber bundles that are common to the whole population of subjects. This method is well-optimized for large intracortical WM projections (Smith et al. 2006), but could be less accurate for thinner cortico-subcortical pathways as the one we identified in our study.

Prior studies have demonstrated that WM structure can influence the distribution of neurostimulatory currents (De Lucia et al. 2007), and correlates with cortico-spinal excitability (Klöppel et al. 2008) and the strength of inter-regional connectivity (Boorman et al. 2007). Our results go however one step further and suggest that interindividual differences in anatomical connectivity patterns between the TMS targeted cortical site and other postsynaptic brain regions are likely to influence the behavioral impact derived from online non-invasive neurostimulation. Due to its trajectory and thinness, a tract between the 
right $\mathrm{FEF}$ and the $\mathrm{SC}$, a structure that in monkeys rivals the striate cortex as the major source of cortico-tectal connectivity (Leichnetz et al. 1981) has yet to be neuroanatomically reported in human brain specimens. Despite the limited intrathalamic resolution of the DTI technique, the anatomical trajectory of our FEF-SC tract strongly suggests that it could correspond to the direct trans-thalamic fronto-tectal tract previously described in monkeys (Leichnetz et al. 1981). These fronto-tectal descending fibers may differently convey the TMS impact in terms of efficiency and speed, due to interindividual tract differences. This fact could explain the observed variability in TMS visual modulations. The SC holds important afferent and efferent connections with posterior parietal regions, the occipital cortex and thalamic nuclei, such as the lateral posterior thalamic complex and the pulvinar (Fries 1984; Robinson \& McClurkin 1989; Sommer \& Wurtz 2000). In addition to its important role in oculomotricity, this midbrain structure is known to be involved in covert attention (Ignashchenkova et al. 2004; Katyal et al. 2010) and visual exploration (Gitelman et al. 2002; Ignashchenkova et al. 2004; Himmelbach et al. 2006). According to some models, interactions between the SC and the pulvinar are considered paramount for mediating attentional influences on perception, under the modulation of cortical regions such as the FEF (Shipp 2004). Additional support for the modulatory role of fronto-tectal connectivity on visual detection comes from studies demonstrating that FEF stimulation influences neuronal activity in the SC of felines (Guitton \& Mandl 1974) and non-human primates (Schlag-Rey et al. 1992), and affects the modulation of saccadic control and the execution of eye movements during visually guided searches. It is thus well known that the SC plays an important role in the detection of visual targets, while driving visually guided eye, head and upper trunk orienting responses towards their location in the extrapersonal space. Nonetheless, its ability to discriminate sophisticated visual objects, faces or natural scenes is more severely curtailed by its rough receptor field organization, and for such tasks, striate visual areas would have to be recruited.

Interestingly, the absence of a significant correlation between any of the four WM tracts and the impact of isolated TMS pulses suggests that the underlying WM system encompasses the combined impact of both the stimulation and valid spatial cues, with additive bearing on visual sensitivity improvements. This notion is further supported by the fact that no significant correlation was found for this same pathway when the behavioral effect of valid spatial cues was considered isolately. Furthermore, FEF TMS pre-target pulse induced significant visual sensitivity modulation only for trials in which attention was effectively oriented towards the location of the upcoming target (Chanes et al. 2012). The contribution of the fronto-tectal pathway is only enabled through the engagement of the endogenous attentional network on a valid location by a predictive cue. Such exquisitively specific influence could be underlied by the state-dependency nature of neurostimulation (Silvanto et al. 2008) suggesting that TMS effects strongly depend on the activation status of the stimulated region. Consequently, TMS activations are likely to rely on the natural ability of such circuitry to facilitate improvements in target detection. These phenomena could be also underlied by cue-triggered reverberating activity across tecto-thalamo-frontal connections, or by tonic patterns of activity within the SC. In support of the latter hypothesis, it has been shown that prior to the execution of a saccade, this tectal region remains in a state of tonically sustained activation, a process that has been associated with its engagement in spatial attentional orienting (Basso \& Wurtz 1998; Glimcher \& Sparks 1992).

Very interestingly, the magnitude of the facilitatory effects on visual detection and the connection strength between the FEF and the SC were significantly anticorrelated. In other words, the higher the fronto-tectal probability of connection, the lower the magnitude of the TMS-driven visual sensitivity increases, and vice-versa. Similarly, negative rather than the expected positive correlations between Fractional Anisotropy (FA) and reaction times for a visuo-spatial task have also been reported elsewhere (Tuch et al 2005). Although this 
explanation remains merely speculative, it could be argued that during the last developmental stages of the central nervous system, a large number of axons linking the FEF and the SC could be pruned out, priming a population of highly selected fibers, which would establish highly excitable connections and induce large postsynaptic potentials in response to weak cortical inputs by neurostimulation. Such pathways could also be more likely denuded from neighboring fibers holding lateral inhibition interactions with the central core of the tract, which might tend to attenuate the extent and weaken connectivitymediated effects. This explanation is consistent with modeling studies proposing that increasing the focality (thus reducing the number of structures activated by the TMS coil) might minimize "parasitic" downstream neurostimulatory effects driven by non-targeted regions, and thus increase its net postsynaptic effects (Wagner et al. 2009). However, this negative correlation could also suggest an inhibitory role for our fronto-tectal pathway. In the latter case, the observed visual facilitatory effects would be mediated by other brain systems, including the right FEF or projections from this area, whereas the FEF-SC pathway could simple act by preventing such visual facilitatory processes from occurring. Finally, we cannot rule out the possibility that such anticorrelation might have been caused by other WM projections not necessarily emerging from the FEF, crossing through the FEF-SC pathway and sustaining a positive correlation with TMS-behavioral effects. Even if this interpretation is theoretically plausible, it would signify that a WM tract not departing from the cortically stimulated area could be more influential than a directly stimulated pathway, an explanation which is not easy to reconcile with observations of network-specific modulations with effects that decay with distance and synaptic chains from the stimulated region (Valero-Cabré et al. 2005; Valero-Cabré et al. 2007).

In the current study, we used probabilistic tractography (also known as "stochastic tractography, see Jones et al. (2012)) to define the white matter pathways from the right FEF, which might have been more likely responsible for TMS driven enhancements of visual facilitation. This method, based on Markov Chain Monte Carlo sampling, models the diffusion of water molecules in each single brain voxel. This approach efficiently simplifies the real motion of molecules in the brain, but it does fail to capture the whole complexity of diffusion in those regions where more than two bundles intersect or in the which the angle between two populations of white matter fibers is below $45^{\circ}$ (Behrens et al. 2007). There may also be additional biases associated with the use of the number of trajectories as an index of connection probability and the favoring of the shortest and the simplest path (Jones 2010), which might not always be the most anatomically realistic and plausible tract between a given origin and a destination in the brain. In consideration of these limitations, the probability of connection reported in our study should be interpreted cautiously and never be taken as a direct measure of the number of axons or as directly correlating with the thickness of their myelin sheaths. Although it is obvious that all these phenomena hold some level of association, the details of such correlates and their covariance remain to be defined. In any case, in accordance with our initial predictions, the FEF-SC pathway which emerged from our correlational study involves the TMS stimulated region with a distant structure such as the $\mathrm{SC}$, whose receptive field organization and processing abilities are compatible with the characteristics (increases of visual detection sensitivity) and also the limitations (lack of effects on visual categorization) of the visual facilitation patterns underlying the correlations of this study (Chanes et al. 2012). In whole fairness however, we cannot rule out that other WM pathways projecting to nodes, which in our study might not have been selected as optimal candidates for correlation, could have also contributed to the observed effects. At least some of the across subject variability encountered could also be underlied by anatomical differences in the precise location of the right FEF by means of average Talairach coordinates and the behavioral consequences of such. 
Furthermore, in this paper we focused in the important but not necessarily unique contribution of WM tracts emerging from the stimulated region. Indeed, it should not be forgotten that other sources of inter-individual variability such as the intrinsic excitability of the stimulated regions and associated networks, the thickness, permittivity and conductivity values of the different tissue layers the magnetic field has to go through, and the relative orientation of neuronal layers with regards to the coil surface, to mention some of them, could also have bearing on the TMS induced current distribution field and its ability to induce neuronal activation (Bijsterbosch et al. 2012). As integrative mapping technologies and more sophisticated human brain-based computational models become available, we will be in position to consider the combined contribution of such variables to TMS effects.

\section{CONCLUSION}

Non-invasive neurostimulation is known to act locally by modulating activity in the stimulated regions of cortex. Nonetheless, its effects can be distantly widespread in a connectivity dependent manner throughout networks departing from the targeted site (Valero Cabré et al. 2005; Valero-Cabré et al. 2007; Wagner et al. 2007 for a review). As a result, an ambiguity prevails with regards to which neural systems under the influence of focal stimulation, alone or in combination with other ongoing activity patterns, might crucially underlie behavioral interferences or ameliorations. By ultimately determining from a set of coherently short-listed WM pathways which ones might best correlate with TMS induced effects, we aimed to decrease the uncertainty on the tracts that might best explain interindividual differences in visual detection facilitatory effects. Significantly correlated pathways could be then considered the most likely associated to the network-wide effects of the neurostimulation on such visual behaviors. Our findings open the door to develop methods that at individual level could help to predict the likeability for neurostimulation to induce a behavioral effect upon the features of the anatomical connections between a neurostimulated cortical site and other interconnected regions. This type of tools and procedures, particularly when supplemented in a near future by additional biophysical and physiological variables which are currently difficult to control for in humans could reveal very useful to evaluate in brain-damaged patients under rehabilitation, the likeability of a relevant therapeutic effect prior to the onset of long neurostimulation regimes.

\section{Acknowledgments}

This work was supported by FP6 (EU VIth Frame Program) \& ANR (Agence National de la Recherche Scientifique) project eraNET-NEURON BEYONDVIS and NINDS (National Institutes of Neurological Disorders and Stroke) R21 NS062317-01A2 granted to AV-C. The participation of RQ was supported by a doctoral fellowship within the eraNET-NEURON BEYONDVIS scheme. The participation of LC was supported by a PhD fellowship of the École des Neurosciences de Paris (ENP). The authors thank Dr. Bruno Dubois for scientific, logistic and financial support to carry out some of the experiments, Drs. Paolo Bartolomeo, Sabine Meunier and Pascale Pradat-Diehl for providing medical supervision during the TMS sessions, Drs. Rushmore, Seth ElkinFrankston, Traian Popa and Claus Hilgetag for fruitful general advice and suggestions on TMS methods and visual and visuo-spatial circuitry, Dr. Michel Thiebaut de Schotten for advice on DTI methods and Dr. Jean Danizeau for help in GLM and statistical matters. We finally thank the coordinators of the IFR neurosciences/CRICM platform and the ER6 UPMC, Drs. Rose Katz, Véronique Marchand-Pauvert and Alexandra Lackmy, for technical and logistic support in the use of TMS equipment.

\section{REFERENCES}

Bartolomeo P, Thiebaut de Schotten M, Chica AB. Brain networks of visuospatial attention and their disruption in visual neglect. Frontiers in human neuroscience. 2012; 6:110. [PubMed: 22586384]

Basser PJ, et al. In vivo fiber tractography using DT-MRI data. Magnetic Resonance in Medicine: Official Journal of the Society of Magnetic Resonance in Medicine / Society of Magnetic Resonance in Medicine. 2000; 44(4):625-632. [PubMed: 11025519] 
Basser PJ, Pierpaoli C. Microstructural and physiological features of tissues elucidated by quantitativediffusion-tensor MRI. Journal of magnetic resonance Series B. 1996; 111(3):209-219. [PubMed: 8661285]

Basso MA, Wurtz RH. Modulation of neuronal activity in superior colliculus by changes in target probability. The Journal of Neuroscience: The Official Journal of the Society for Neuroscience. 1998; 18(18):7519-7534. [PubMed: 9736670]

Beauchamp MS, et al. A Parametric fMRI Study of Overt and Covert Shifts of Visuospatial Attention. NeuroImage. 2001; 14(2):310-321. [PubMed: 11467905]

Behrens TEJ, et al. Probabilistic diffusion tractography with multiple fibre orientations: What can we gain? NeuroImage. 2007; 34(1):144-155. [PubMed: 17070705]

Bijsterbosch JD, et al. Where does transcranial magnetic stimulation (TMS) stimulate? Modelling of induced field maps for some common cortical and cerebellar targets. Medical \& biological engineering \& computing. 2012; 50(7):671-681. [PubMed: 22678596]

Boorman ED, et al. Individual differences in white-matter microstructure reflect variation in functional connectivity during choice. Current Biology: CB. 2007; 17(16):1426-1431. [PubMed: 17689962]

Cameron EL, Tai JC, Carrasco M. Covert attention affects the psychometric function of contrast sensitivity. Vision Research. 2002; 42(8):949-967. [PubMed: 11934448]

Carrasco M, P.Talgar C, Cameron EL. Characterizing visual performance fields: effects of transient covert attention, spatial frequency, eccentricity, task and set size. Spatial Vision. 2001; 15:61-75. [PubMed: 11893125]

Carrasco M, Penpeci-Talgar C, Eckstein M. Spatial covert attention increases contrast sensitivity across the CSF: support for signal enhancement. Vision Research. 2000; 40(10-12):1203-1215. [PubMed: 10788636]

Chica AB, Bartolomeo P, Valero-Cabré A. Dorsal and Ventral Parietal Contributions to Spatial Orienting in the Human Brain. The Journal of Neuroscience. 2011; 31(22):8143-8149. [PubMed: 21632936]

Corbetta M, Shulman GL. Control of goal-directed and stimulus-driven attention in the brain. Nat Rev Neurosci. 2002; 3(3):201-215. [PubMed: 11994752]

Ekstrom LB, et al. Modulation of the Contrast Response Function by Electrical Microstimulation of the Macaque Frontal Eye Field. The Journal of Neuroscience. 2009; 29(34):10683-10694. [PubMed: 19710320]

Fries W. Cortical projections to the superior colliculus in the macaque monkey: a retrograde study using horseradish peroxidase. The Journal of Comparative Neurology. 1984; 230(1):55-76. [PubMed: 6096414]

Gattass R, Desimone R. Responses of cells in the superior colliculus during performance of a spatial attention task in the macaque. Revista Brasileira De Biologia. 1996; 56(Su 1)(Pt 2):257-279. [PubMed: 9394506]

Gitelman, Darren R., et al. Functional anatomy of visual search: regional segregations within the frontal eye fields and effective connectivity of the superior colliculus. NeuroImage. 2002; 15(4): 970-982. [PubMed: 11906237]

Glimcher PW, Sparks DL. Movement selection in advance of action in the superior colliculus. Nature. 1992; 355(6360):542-545. [PubMed: 1741032]

Groppe DM, Urbach TP, Kutas M. Mass univariate analysis of event-related brain potentials/fields I: a critical tutorial review. Psychophysiology. 2011; 48(12):1711-1725. [PubMed: 21895683]

Grosbras M, Paus Tomáš. Transcranial magnetic stimulation of the human frontal eye field facilitates visual awareness. European Journal of Neuroscience. 2003; 18(11):3121-3126. [PubMed: 14656308]

Grosbras M-H, Paus Tomáš. Transcranial Magnetic Stimulation of the Human Frontal Eye Field: Effects on Visual Perception and Attention. Journal of Cognitive Neuroscience. 2011; 14(7):11091120. [PubMed: 12419133]

Guitton D, Mandl G. The effect of frontal eye field stimulation on unit activity in the superior colliculus of the cat. Brain Research. 1974; 68(2):330-334. [PubMed: 4826902] 
Hilgetag CC, Theoret H, Pascual-Leone A. Enhanced visual spatial attention ipsilateral to rTMSinduced «virtual lesions » of human parietal cortex. Nat Neurosci. 2001; 4(9):953-957. [PubMed: 11528429]

Himmelbach M, Erb M, Karnath H-O. Exploring the visual world: The neural substrate of spatial orienting. NeuroImage. 2006; 32(4):1747-1759. [PubMed: 16806986]

Huerta MF, Krubitzer LA, Kaas JH. Frontal eye field as defined by intracortical microstimulation in squirrel monkeys, owl monkeys, and macaque monkeys. II. Cortical connections. The Journal of comparative neurology. 1987; 265(3):332-361. [PubMed: 2447132]

Ignashchenkova A, et al. Neuron-specific contribution of the superior colliculus to overt and covert shifts of attention. Nature Neuroscience. 2004; 7(1):56-64.

Jones DK. Challenges and limitations of quantifying brain connectivity in vivo with diffusion MRI. Imaging in Medicine. 2010; 2(3):341-355.

Jones DK, Knösche TR, Turner R. White matter integrity, fiber count, and other fallacies: The do's and don'ts of diffusion MRI. NeuroImage. 2012

Katyal S, et al. Topography of Covert Visual Attention in Human Superior Colliculus. Journal of Neurophysiology. 2010; 104(6):3074-3083. [PubMed: 20861435]

Kincade JM, et al. An Event-Related Functional Magnetic Resonance Imaging Study of Voluntary and Stimulus-Driven Orienting of Attention. The Journal of Neuroscience. 2005; 25(18):4593-4604. [PubMed: 15872107]

Klöppel S, et al. The cortical motor threshold reflects microstructural properties of cerebral white matter. NeuroImage. 2008; 40(4):1782-1791. [PubMed: 18342540]

Leichnetz GR, et al. The prefrontal corticotectal projection in the monkey; An anterograde and retrograde horseradish peroxidase study. Neuroscience. 1981; 6(6):1023-1041. [PubMed: 6168970]

De Lucia M, et al. Diffusion tensor MRI-based estimation of the influence of brain tissue anisotropy on the effects of transcranial magnetic stimulation. NeuroImage. 2007; 36(4):1159-1170. [PubMed: 17524673]

Michel, Thiebaut de Schotten, et al. A Lateralized Brain Network for Visuo-Spatial Attention. 2011. Available at: http://precedings.nature.com/documents/5549/version/1 [Consulté le mars 2, 2011].

Moore T, Fallah M. Microstimulation of the Frontal Eye Field and Its Effects on Covert Spatial Attention. Journal of Neurophysiology. 2004; 91(1):152-162. [PubMed: 13679398]

Nobre AC, et al. Functional localization of the system for visuospatial attention using positron emission tomography. Brain: a journal of neurology. 1997; 120(Pt 3):515-533. [PubMed: 9126062]

O'Connor DH, et al. Attention modulates responses in the human lateral geniculate nucleus. Nat Neurosci. 2002; 5(11):1203-1209. [PubMed: 12379861]

Paus, Tomás. Location and function of the human frontal eye-field: A selective review. Neuropsychologia. 1996; 34(6):475-483. [PubMed: 8736560]

Petit L, et al. Functional Asymmetries Revealed in Visually Guided Saccades: An fMRI Study. Journal of Neurophysiology. 2009; 102(5):2994-3003. [PubMed: 19710382]

Reynolds JH, Chelazzi L. ATTENTIONAL MODULATION OF VISUAL PROCESSING. Annual Review of Neuroscience. 2004; 27(1):611-647.

Reynolds JH, Desimone Robert. Interacting Roles of Attention and Visual Salience in V4. Neuron. 2003; 37(5):853-863. [PubMed: 12628175]

Reynolds JH, Pasternak T, Desimone Robert. Attention Increases Sensitivity of V4 Neurons. Neuron. 2000; 26(3):703-714. [PubMed: 10896165]

Robinson DL, McClurkin JW. The visual superior colliculus and pulvinar. Reviews of Oculomotor Research. 1989; 3:337-360. [PubMed: 2486329]

Ruff CC, et al. Concurrent TMS-fMRI and psychophysics reveal frontal influences on human retinotopic visual cortex. Current Biology: CB. 2006; 16(15):1479-1488. [PubMed: 16890523]

Schlag-Rey M, Schlag J, Dassonville P. How the frontal eye field can impose a saccade goal on superior colliculus neurons. Journal of Neurophysiology. 1992; 67(4):1003-1005. [PubMed: 1588383] 
Schneider KA, Kastner S. Effects of Sustained Spatial Attention in the Human Lateral Geniculate Nucleus and Superior Colliculus. The Journal of Neuroscience. 2009; 29(6):1784-1795. [PubMed: 19211885]

Shipp S. The brain circuitry of attention. Trends in Cognitive Sciences. 2004; 8(5):223-230. [PubMed: 15120681]

Shulman GL, et al. Right Hemisphere Dominance during Spatial Selective Attention and Target Detection Occurs Outside the Dorsal Frontoparietal Network. The Journal of Neuroscience. 2010; 30(10):3640-3651. [PubMed: 20219998]

Silvanto J, Lavie N, Walsh Vincent. Stimulation of the Human Frontal Eye Fields Modulates Sensitivity of Extrastriate Visual Cortex. Journal of Neurophysiology. 2006; 96(2):941-945. [PubMed: 16624999]

Silvanto J, Muggleton N, Walsh Vincent. State-dependency in brain stimulation studies of perception and cognition. Trends in cognitive sciences. 2008; 12(12):447-454. [PubMed: 18951833]

Smith SM, et al. Tract-based spatial statistics: voxelwise analysis of multi-subject diffusion data. NeuroImage. 2006; 31(4):1487-1505. [PubMed: 16624579]

Sommer MA, Wurtz Robert H. Composition and Topographic Organization of Signals Sent From the Frontal Eye Field to the Superior Colliculus. Journal of Neurophysiology. 2000; 83(4):1979-2001. [PubMed: 10758109]

Stanton GB, Bruce CJ, Goldberg ME. Topography of projections to posterior cortical areas from the macaque frontal eye fields. The Journal of comparative neurology. 1995; 353(2):291-305. [PubMed: 7745137]

Thiebaut de Schotten M, et al. Monkey to human comparative anatomy of the frontal lobe association tracts. Cortex; a journal devoted to the study of the nervous system and behavior. 2012 ; 48(1):8296.

Thielscher A, Kammer T. Electric field properties of two commercial figure- 8 coils in TMS: calculation of focality and efficiency. Clinical Neurophysiology. 2004; 115(7):1697-1708. [PubMed: 15203072]

Thut G, Nietzel A, Pascual-Leone A. Dorsal Posterior Parietal rTMS Affects Voluntary Orienting of Visuospatial Attention. Cerebral Cortex. 2005; 15(5):628-638. [PubMed: 15342434]

Umarova RM, et al. Structural Connectivity for Visuospatial Attention: Significance of Ventral Pathways. Cerebral Cortex. 2010; 20(1):121-129. [PubMed: 19406904]

Valero-Cabré A, et al. Impact of repetitive transcranial magnetic stimulation of the parietal cortex on metabolic brain activity: a 14C-2DG tracing study in the cat. Experimental Brain Research. 2005; 163(1):1-12.

Valero-Cabré A, Payne BR, Pascual-Leone A. Opposite impact on 14C-2-deoxyglucose brain metabolism following patterns of high and low frequency repetitive transcranial magnetic stimulation in the posterior parietal cortex. Experimental Brain Research. Experimentelle HirnforschungExpérimentation Cérébrale. 2007; 176(4):603-615.

Wagner, Tim, et al. Biophysical foundations underlying TMS: Setting the stage for an effective use of neurostimulation in the cognitive neurosciences. Cortex. 2009; 45(9):1025-1034. [PubMed: 19027896]

Wagner, Timothy; Valero-Cabre, A.; Pascual-Leone, A. Noninvasive human brain stimulation. Annual Review of Biomedical Engineering. 2007; 9:527-565.

Wurtz RH, Goldberg ME. Activity of superior colliculus in behaving monkey. IV. Effects of lesions on eye movements. Journal of Neurophysiology. 1972; 35(4):587-596. [PubMed: 4624742]

Yeshurun Y, Carrasco M. Spatial attention improves performance in spatial resolution tasks. Vision Research. 1999; 39(2):293-306. [PubMed: 10326137] 


\section{Highlights}

- Fronto-tectal connectivity and detection performance are significantly correlated

- Fronto-Tectal connectivity influences the effects of FEF TMS on visual detection

- $\quad$ TMS and WM interindividual differences help to disambiguate mediating anatomical pathways

- This approach could be useful to predict the efficiency of focal neurostimulation 
a
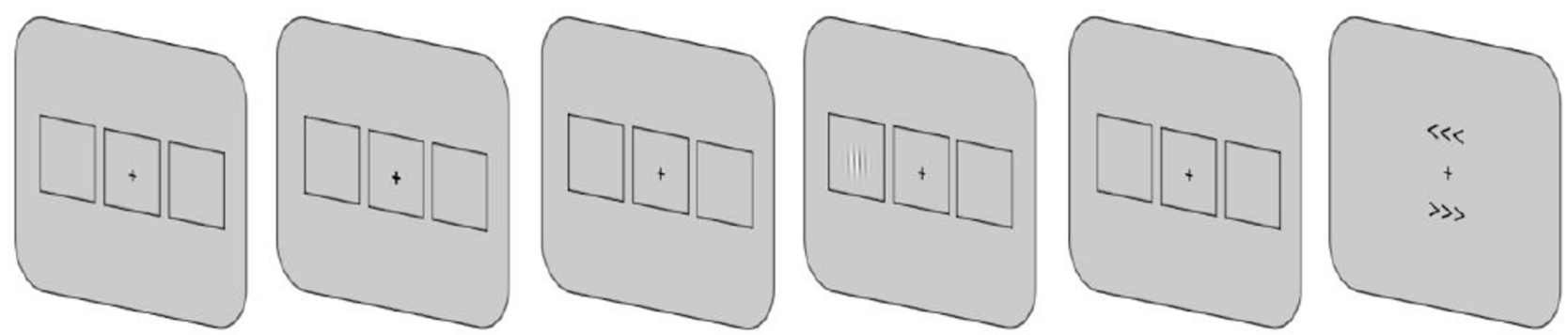

Time

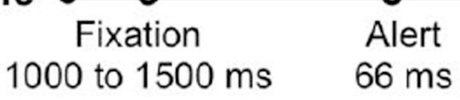

ISI

$233 \mathrm{~ms}$

$+80 \mathrm{~ms}$ pre-target

Target

$33 \mathrm{~ms}$

Categorization

Detection TMS

b
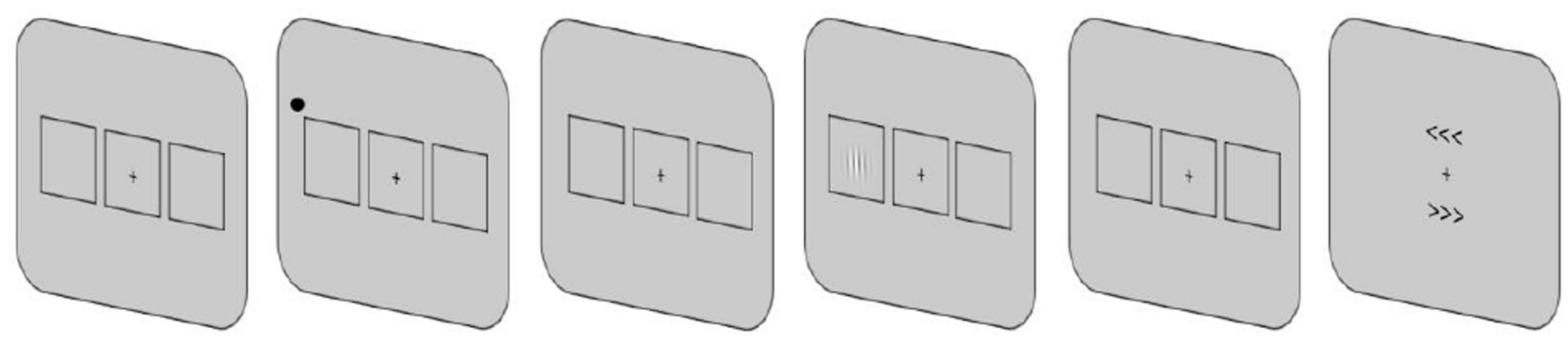

Time

Fixation

Cue

$66 \mathrm{~ms}$

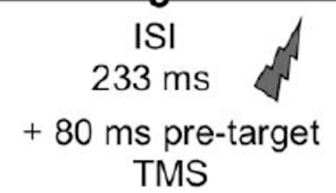

Target

$33 \mathrm{~ms}$

Categorization

Detection

1000 to $1500 \mathrm{~ms}$

TMS

Figure 1.

Sequence of events during a representative trial for each of the TMS Experiments, whose data sets were used in our study. In both, participants were requested to fixate at a central cross for a randomly variable period of time between 1000 to $1500 \mathrm{~ms}$. In experiment 1 (a), there was no peripheral cue but the central cross became slightly bigger for $66 \mathrm{~ms}$ to alert participants of an upcoming event. The TMS pulse was delivered on the right FEF 80, 100 or $140 \mathrm{~ms}$ prior to target onset. In experiment 2 (b), a peripheral visuo-spatial cue, consisting in a black circle was displayed for $66 \mathrm{~ms}$ to the right or the left of the fixation cross. The cue was predictive about the location of the subsequent target (75\% valid and $25 \%$ invalid trials), and was followed by a TMS pulse delivered $80 \mathrm{~ms}$ pre-target onset. In both experiments, active or sham TMS pulses were interleaved in a randomized order. Then, after an interstimulus interval (ISI) of $233 \mathrm{~ms}$, a Gabor with the lines tilted to the left or the right appeared for $33 \mathrm{~ms}$ at the center of one of the two lateral boxes. Participants were then requested to perform two sequential tasks; first a visual categorization task to indicate the orientation of the Gabor lines (left/right) and second, a conscious visual detection task in which they had to report if they did see the target, and where they saw it (left/right). A cue was considered valid when it correctly signaled the location of the upcoming target (left or right), and invalid when it incorrectly signaled target location. A valid trial was the one including a valid cue whereas the opposite applies to invalid trials. The lower panel (b) shows for Experiment 2 an example of a valid trial (see Chanes et al. 2012 for full details on the behavioral paradigms). 


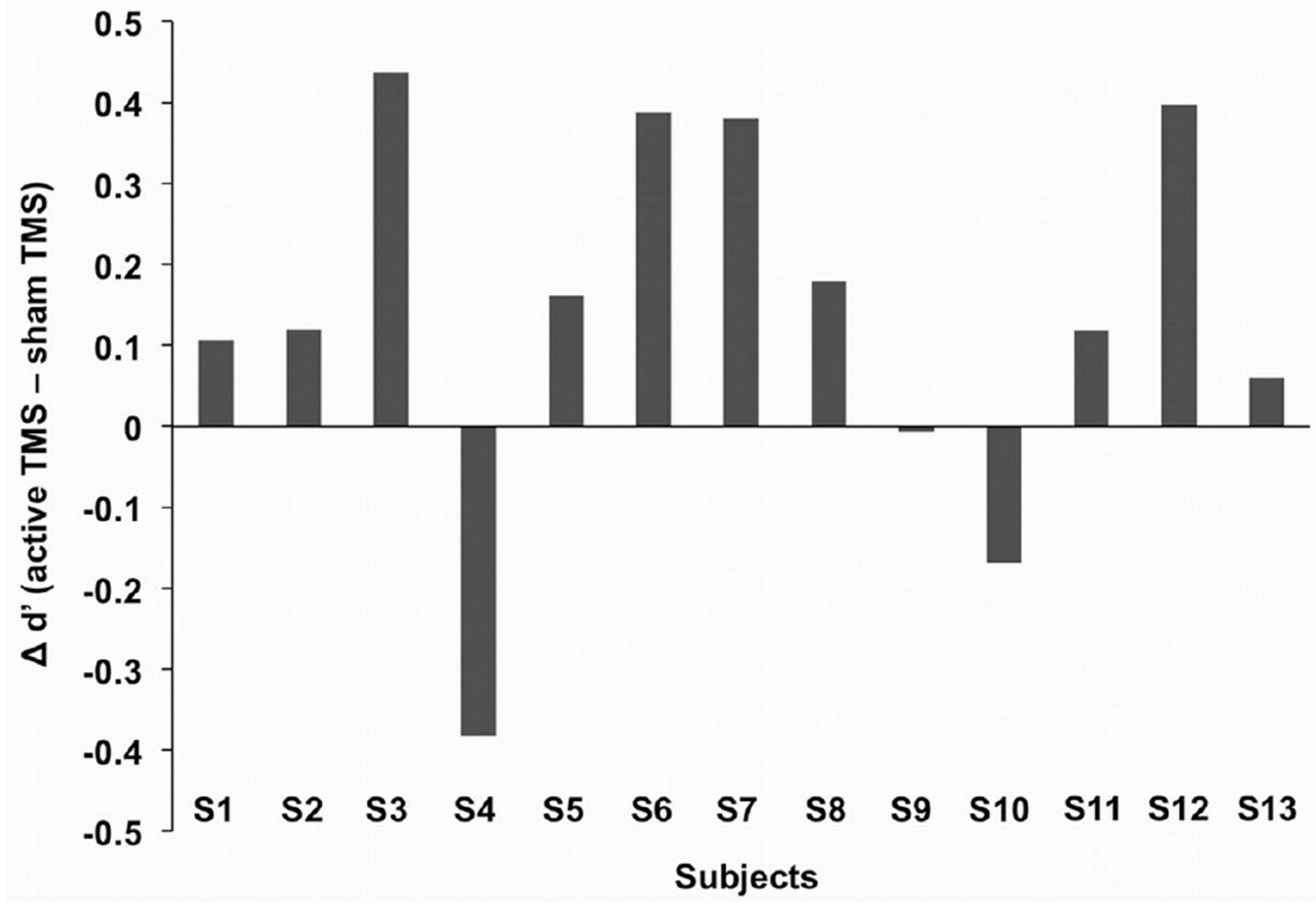

Figure 2.

Individual behavioral data displaying visual detection sensitivity differences $(\Delta d$ ') induced by the effects of TMS (active TMS - sham TMS) for each of the 13 participants (S1 to S13) in the study. Positive values indicate detection sensitivity increases, hence better visual detection performance under real than sham TMS. Negative values indicate detection sensitivity decreases, and thus worse detection performance under real than sham TMS. Notice that even if as a group, statistically significantly improvements of visual sensitivity under real TMS were found for those participants who correctly oriented their attention in response to valid spatial cues (Chanes et al. 2012), there was a degree of behavioral variability in the direction and magnitude of visual performance effects across the population of 13 participants who were included in the current study. 

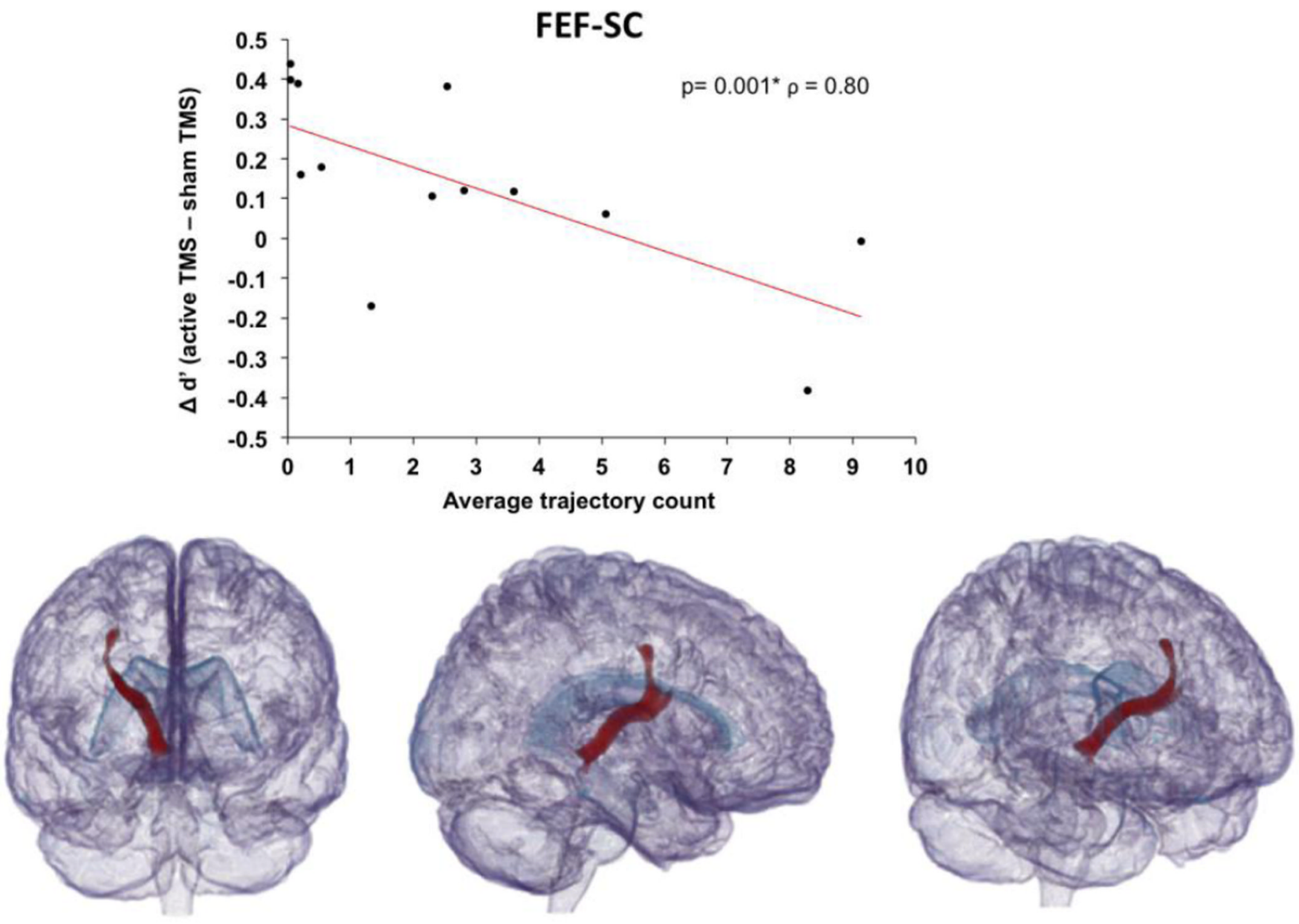

Figure 3.

Correlation plot (top) representing the magnitude of the TMS driven modulations in visual sensitivity ( $\Delta \mathrm{d}^{\prime}$ ) (active TMS - sham TMS) for the detection of validly cued visual targets (Chanes et al. 2012), crossed with the average trajectory counts between the right FEF and the ipsilateral SC according to diffusion data. See (bottom) three dimensional glass brain views (left to right: front view, side view, and 3/4 quarter rear right view) represented in MNI standardized space, corresponding to the mean FEF-SC tract recorded in our population of 13 participants. Notice that it was only for this tract that our data revealed a statistically significant correlation between the average trajectory counts between the FEF and the SC and the modulation of visual perceptual sensitivity (active TMS - sham TMS). By dividing the average trajectory count values by the number of trajectories launched from each seed voxel, i.e. 5000, a probability of connection between 0 and 1 can be calculated. 

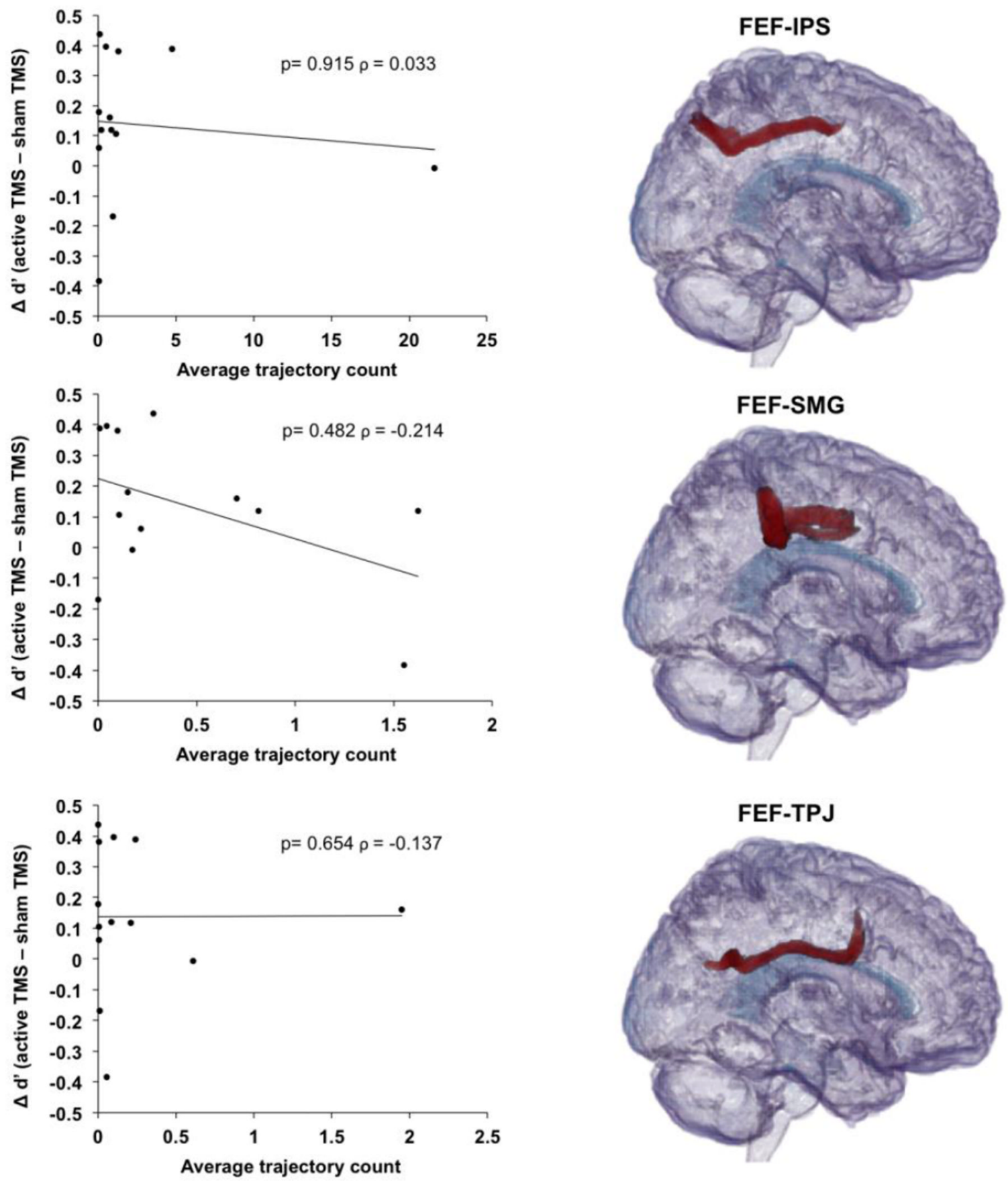

Figure 4.

Correlation plots representing the magnitude of the TMS driven modulations on visual sensitivity ( $\Delta \mathrm{d}^{\prime}$ ) (active TMS - sham TMS) for the detection of validly cued visual targets (Chanes et al. 2012), crossed with the average trajectory counts between the right FEF and the ipsilateral IPS, SMG and TPJ sites (left panels, from top to bottom) respectively. For each of these three tracts (FEF-IPS, FEF-SMG, FEF-TPJ) we present (right panels, from top to bottom) dimensional glass brain selected views in MNI standardized space of the mean white matter tract for the 13 participants of the study. Notice that for none of those three tracts there was any statistically significant correlation between the average trajectory counts and the modulation of visual perceptual sensitivity (active TMS - sham TMS). By dividing 
the average trajectory count values by the number of trajectories launched from each seed voxel, i.e. 5000, a probability of connection between 0 and 1 can be calculated. 


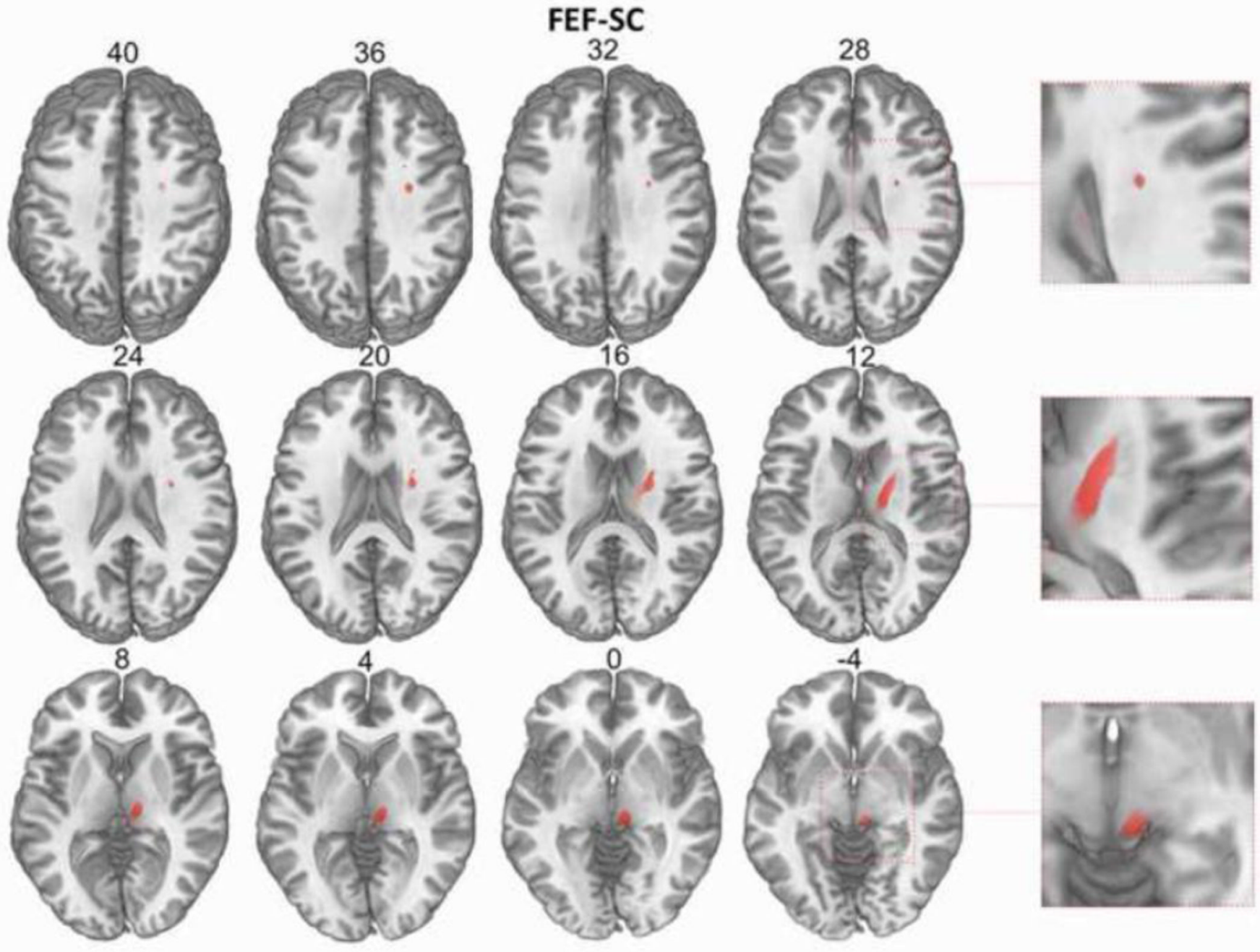

Figure 5.

Mean anatomical trajectory within a brain template of the most probable connection between the FEF and the SC revealed by our study, found correlated with visual sensitivity modulations. The average fronto-tectal pathway from the 13 subjects of the study is presented in MNI standardized space across serial selected axial sections (see $\mathrm{z}$-coordinates) organized from top (FEF) to bottom (SC). Notice the progression of this tract through the corona radiata underlying the FEF in the right hemisphere, the internal capsule, and the right posterior thalamus to finally reach the superior colliculus in the upper tectum. The anatomical trajectory of this FEF-SC tract strongly suggests that it could correspond to the trans-thalamic fronto-tectal tract previously reported in monkeys (Leichnetz et al. 1981). 